\title{
Accountable Care Organizations: What Radiologists Should Know
}

R.A. Charalel and P.C. Sanelli

ABBREVIATION: $\mathrm{ACO}=$ Accountable Care Organization

$\mathbf{T}$ he landscape of health care is quickly changing, with new legislation and economic factors driving health care reform. Yet, fundamentally many health care delivery professionals, including physicians, remain unaware of the changing landscape and its implications for their practice. We seek to review Accountable Care Organizations (ACOs), a legal entity developed in November 2011, and their implications for radiologists.

\section{WHY WERE ACCOUNTABLE CARE ORGANIZATIONS CREATED?}

ACOs were created in an amendment to the Patient Protection and Affordable Care Act to foster shared savings between Medicare and health care providers. By eliminating unnecessary procedures and tests, ACOs strive to reduce overall health care expenditures, improve health care outcomes, and redistribute a portion of the shared savings between the provider and Medicare.

\section{WHAT IS AN ACO?}

ACOs are groups of physicians, hospitals, and other health care workers who come together by choice to provide high-quality health care to Medicare patients. Four main subtypes of ACOs exist, including the Medicare Shared Savings Program, the Advance Payment ACO Model, the Pioneer ACO Model, and ACO: Accelerated Development Learning Sessions. The Medicare Shared Savings Program is targeted toward providers who provide fee-for-service health care to Medicare patients or beneficiaries. ${ }^{1}$ This program is incentivized to reduce health care costs by allowing ACO participants to split shared savings, which are determined by the annual decrease in health care costs compared with an individual ACO's calculated benchmark (on the basis of prior 3-year spending) and specific contract terms. The Advance

Received August 6, 2013; accepted after revision August 8.

From the Department of Radiology, New York-Presbyterian Hospital/Weill Cornell Medical Center, New York, New York.

Please address correspondence to Pina C. Sanelli, MPH, FACR, 1305 York Ave, 3rd Floor, New York, NY 10021; e-mail: pcs9001@med.cornell.edu

http://dx.doi.org/10.3174/ajnr.A3771
Payment ACO Model is a supplementary incentive program for eligible providers already participating in the Medicare Shared Savings Program. The Pioneer ACO Model is intended for health care organizations and providers already experienced in coordinating care for patients across care settings. For existing or emerging ACOs, there is also an opportunity to understand population health while reducing cost growth as well as how to establish and implement care delivery through the ACO: Accelerated Development Learning Sessions. For providers who are interested in newly joining an ACO, the Medicare Shared Savings Program is currently accepting applications. ${ }^{1}$

\section{WHAT ARE THE REQUIREMENTS FOR ELIGIBILITY/PARTICIPATION?}

To form an ACO, a provider must be Medicare-enrolled and bill for primary care services (as defined by Medicare billing codes). ACOs must have a group of Medicare patients (who are termed beneficiaries), for which the single ACO provides all primary care. Providers may join an ACO as an ACO participant or other entity. ACO participants must have a 3-year Medicare billing history, with a Medicare-enrolled taxpayer identification number, to calculate a benchmark and properly assign beneficiaries. ${ }^{1}$ If a provider does meet ACO participant eligibility criteria or wishes to have more flexibility, they may join an ACO as another entity. Another entity is simply a Medicare-enrolled group that bills Medicare through a Medicare-enrolled taxpayer identification number. If provider(s) do not have a 3-year history of billing for primary care services, they may join with ACO participant(s) who have an eligible history and form an ACO together. Once an ACO is formed, the contract is valid for 3 years, though there is opportunity for addition or removal of ACO participants annually. ${ }^{1}$

\section{HOW CAN A RADIOLOGIST PARTICIPATE?}

In addition to providing image interpretation, radiologists may contribute to cost savings programs by guiding imaging examination choices ${ }^{2}$ on the basis of evidence-based guidelines (such as the American College of Radiology appropriateness criteria) and 
local practice patterns for standard of care. Radiologists are uniquely qualified to serve in such a role, given their imaging expertise, and would add value to the ACO system by decreasing the number of unnecessary imaging examinations and helping to provide the patient with the best possible examination.

As a specialist, a radiologist may become involved as an ACO participant or as another entity. However, shared savings are calculated through the use of only contributions from ACO participants and are thus only distributed among ACO participants. Groups that join an ACO as another entity are not eligible to profit from shared savings. In addition, ACO participants who are not billing for primary care services are eligible to be a participant in more than one ACO. As a result, for radiologists who are billing for specialized services (not primary care), they may be able to team up with a number of different ACOs and benefit from shared savings in multiple groups.

\section{WHAT ARE THE CHALLENGES?}

Even in an initially ideal scenario with increased shared savings, given reduced numbers of unnecessary imaging procedures, radiologists may face potentially negative downstream effects. Initially radiologists may expect decreased demand for imaging procedures and make up some of this reduced revenue with the shared savings that this cost reduction may create. However, as spending continues to decrease and the benchmark cost (on the basis of prior 3-year spending) also decreases, the practice may cease to be able to further reduce costs and thus be ineligible for future savings after the first few successful years.

Additionally, the logistics of providing a gateway for study approval are challenging and may have a number of new implications. ${ }^{2}$ First, there is potential to be penalized for increased numbers of performed imaging examinations as compared with a national or local average, despite the reality that the decision to perform a study is joint between radiologist and clinician, and does not rest solely with the radiologist. Second, if a radiologist is not compliant with a study, the radiologist may be implicated in any potential downstream tort cases, despite an informed decision by both clinician and patient. In such a situation, formalizing the role of image selection consultation, by use of evidence-based criteria, may actually protect radiologists, given that it will provide the opportunity to document rationale.

Another challenge to ACO health care delivery is that effective teams, consisting of health care providers from different special- ties, are essential for synchronizing and coordinating care efficiently. ${ }^{3}$ To be successful, health care providers in an ACO will need to utilize specific skills in the areas of collaboration, communication, and teamwork to coordinate care ${ }^{4}$ that will not only reduce cost but also improve outcomes.

\section{WHAT ARE THE FUTURE IMPLICATIONS?}

Although initially, it may seem challenging to redistribute health care delivery systems to create an ACO, there are many incentives to do so. First, there is the promise of potential shared savings by reducing unnecessary imaging examinations and procedures while still delivering high-quality health care. Second, in their current stage, ACOs are entirely voluntary groups that come together to accomplish a shared goal. At this stage, the terms of an ACO are still malleable. In the future, such groups may have rigid structures with committees and chairs, which may be difficult for radiologists to join or have a significant voice within. As a result, it is advantageous to participate in ACOs and increase our bargaining power within them while the structure is not yet predetermined. Finally, there are a number of ramifications to being left behind, aside from losing shared savings. Groups who do not participate in ACOs may be viewed as non-resource conscious and penalized in future legislation. Additionally, they may lose opportunities to grow their practice referrals or gain leadership experience in imaging examination selection and ultimately may be viewed as less desirable ACO participants in the future if forced to participate. Thus, although there is considerable angst and reluctance to join ACOs, it may be beneficial to get involved early on, so that we can actively help shape the changing landscape of health care.

\section{REFERENCES}

1. Medicare Shared Savings Program. Frequently asked questions. http://www.cms.gov/Medicare/Medicare-Fee-for-Service-Payment/ sharedsavingsprogram/Downloads/MSSP-FAQs.pdf. Accessed June 15,2013

2. Breslau J, Lexa FJ. A radiologist's primer on accountable care organizations. J Am Coll Radiol 2011;8:164-68

3. McClellan M, McKethan AN, Lewis JL, et al. A natural strategy to put accountable care into practice. Health Affairs 2010;29:982-90

4. Press MJ, Michelow MD, MacPhail LH. Care coordination in accountable care organizations: moving beyond structure and incentives. Am J Manag Care 2012;18:778 -80 